\title{
El nivel de la calidad de gasto público del proceso presupuestario en la dirección regional de transportes y comunicaciones de San Martín, 2019
}

\author{
Janeth Pinedo García \\ janeth-2207@hotmail.com \\ Facultad de Ciencias Empresariales \\ Universidad Peruana Unión \\ Tarapoto, Perú \\ ORCID: 0000-0002-3690-9318 \\ Johnny Dávila Ruiz \\ johnnydruiz1975@gmail.com \\ Facultad de Ciencias Empresariales \\ Universidad Peruana Unión \\ Tarapoto, Perú \\ Edison Eli Luna Risco \\ elunarisco@gmail.com \\ Facultad de Ciencias Empresariales \\ Universidad Peruana Unión \\ Tarapoto, Perú
}

\section{RESUMEN}

El objetivo fue medir el nivel de la calidad de gasto público respecto al proceso presupuestario en la Dirección Regional de Transportes y Comunicaciones de San Martin durante el año 2019, el cual fue deficiente debido que el avance de ejecución presupuestal fue de $81.1 \%$. El diseño de investigación fue no experimental de tipo documental, se usó la ficha de registro documental como instrumento de recojo de información, teniendo como base documentos confiables de fuentes primarias y secundarias. Los resultados mostraron que la formulación presupuestal se definió en base a 51 metas físicas, de las cuales la meta 04 fue la más significativa con un monto de S/ 4985 381.00. La aprobación del proceso presupuestario total inició con un PIA de S/. 14265 378.00, el cual derivó en un PIM de S/. 16896 440.00. En la ejecución presupuestal durante el periodo 2019, los ingresos por recursos directamente recaudados fueron de S/. 3091 930.18, asimismo la ejecución de los gastos atendidos por los recursos ordinarios fue del 95.03\%, en los recursos directamente recaudados se ejecutó el $47.45 \%$ y por recursos operaciones oficiales de crédito fue de $0 \%$.

Palabras clave: gasto público; proceso presupuestario; calidad de gasto; presupuesto público. 


\title{
Quality level of public expenditure of the budgetary process in the regional directorate of transport and communications of san martin, 2019
}

\begin{abstract}
:
The objective was to measure the level of the quality of public spending with respect to the budget process in the Regional Directorate of Transport and Communications of San Martin during 2019, which was deficient due to the progress of budget execution was $81.1 \%$. The research design was non-experimental of the documentary type, the document record sheet was used as an instrument for collecting information, based on reliable documents from primary and secondary sources. The results showed that the budget formulation was defined based on 51 physical goals, of which goal 04 was the most significant with an amount of S / 4,985,381.00. The approval of the total budget process began with a PIA of S /. 14265 378.00, which resulted in a PIM of S /. 16896 440.00. In the budget execution during the 2019 period, the income from resources directly collected was S /. 3091930.18 , likewise the execution of the expenses attended by ordinary resources was $95.03 \%$, in the resources directly collected $47.45 \%$ was executed and by resources official credit operations was $0 \%$.
\end{abstract}

Keywords: Public spending, budget process, quality of spending, public budget

Artículo recibido: 02 noviembre. 2021 Aceptado para publicación: 28 noviembre 2021 Correspondencia: janeth-2207@ hotmail.com Conflictos de Interés: Ninguna que declarar 


\section{INTRODUCCIÓN}

A nivel internacional se reconoce que la calidad de gasto está referida a aquellas acciones en las que se incurrieron gastos públicos de modo eficiente, para lo cual es sumamente indispensable que se cumplan ciertos lineamientos y normativas que buscan contribuir con el crecimiento y desarrollo de nuestro país y, a su vez, cubrir las necesidades y requerimientos de la ciudadanía (Guerra y Palomino, 2020). Asimismo, cabe mencionar que todas las entidades estatales y organismos gubernamentales disponen de un presupuesto asignado para ejecutar sus gastos, los mismos que deben estar consignados para la mejora de sus infraestructuras, salud pública, seguridad pública, etc. (Galiya et al., 2018). Sin embargo, actualmente existe una clara concordancia, de que la administración pública es prestadora de gran insatisfacción, por su actuar definido por servicios o procesos lentos, caros, corruptos, de baja o mala calidad; que además de estar mal diseñados, no se centran, ni mucho menos cubren las necesidades más apremiantes de la población, sino que también, como si no bastara con ello, se producen despilfarros de recursos humanos y financieros, que indignan a todos (Vargas y Zavaleta, 2020). Todas esta acciones están presente en muchas entidades públicas de los países, que se desvían del objetivo principal de la gestión presupuestaria el cual tiene como fin permanente la búsqueda de incrementar la productividad de las actividades y la utilización eficiente y racional de los limitados recursos económicos, así como al derecho que tiene el usuario final de recibir una atención satisfactoria que contribuya a mejorar su calidad de vida (Yactayo, 2019). Aunado a ello, las instituciones públicas carecen de buen gobierno, presentando muchas fuentes de defectos que degeneran la administración pública, configurándose como ineficiente, corrupta, derrochadora de recursos, que utiliza los compadrazgos por encima de la idoneidad para el cabal cumplimiento de la función pública (Schröder, 2013).

De manera análoga, en el Perú muchas entidades públicas, tienen modelos de gestión presupuestaria, según normas y procedimientos vigentes que regulan sus actividades, no obstante, la operatividad de la fase de ejecución presupuestal no está articulada con la producción de las metas y su gasto público del proceso presupuestario no lo realizan con buena eficiencia e idoneidad (Yactayo, 2019). Además, en el Perú, existe un significativo distanciamiento entre la formulación, diseño e implementación de políticas y los resultados que se obtienen de ellas, llevando a cabo muchas veces una mala ejecución del 
presupuesto público, olvidando que el presupuesto es la herramienta principal que tiene el gobierno para obtener buenos resultados de sus intervenciones en términos calidad, costo, eficiencia, efectividad, equidad y oportunidad; (Comisión Económica para América Latina y el Caribe, 2002). Consecuencia de ello el Ejecutivo en el 2019, presentó uno de sus peores registros en ejecución presupuestal, ya que tuvo más de 2,500 proyectos pendientes, a pesar de que cada ministerio contó con el presupuesto correspondiente para cumplir con sus objetivos (Carmen Moran, 2019). También la ejecución presupuestal al cierre del año 2019 en el país, en la categoría presupuestaria Programas Presupuestales se registró 89 Programas Presupuestales que se establecieron para el año fiscal, de los cuales 22 de ellos registraron una ejecución alta (superior a 95.3\%). A su vez, 45 Programas Presupuestales, mostraron una ejecución moderada (entre 78.9\% a 95.3\%); mientras que 22 Programas restantes registraron una ejecución baja (inferior a 78.9\%); de los programas con ejecución baja, se ubicaron los Programas Presupuestales adheridos al sector transporte, donde el programa presupuestal de reducción del costo, tiempo e inseguridad en el sistema de transporte tuvo ejecución de $74.5 \%$ y el programa presupuestal reducción del tiempo, inseguridad y costo ambiental en el transporte urbano tuvo la ejecución de $64.7 \%$, reflejando total ineficiencia en este ámbito el cual es preocupante para el país (Ministerio de Economía y Finanzas, 2020). Dichos resultados son provenientes, de la corrupción y la inconducta funcional a nivel de gobierno, en donde los sectores de transporte y comunicaciones, salud y educación han tenido una pérdida de ejecución presupuestal alrededor del 15\% (Shack et al., 2020).

En otra parte, el Gobierno Regional de San Martín en el periodo 2019 según la consulta amigable del Ministerio de Economía y Finanzas, la ejecución presupuestal se ubica en la sexta posición entre los 25 gobiernos regionales del país, con un $90.8 \%$ de avance, de un PIM 1559 891 831. Sin embargo, entre ellos la Unidad Ejecutora 200-925: Región san Martin-Transportes o Dirección Regional de Transportes y Comunicaciones de San Martín, como parte del Gobierno Regional de San Martin, tuvo problemas en la ejecución presupuestal, lo que repercutió en la calidad de gasto en todas sus categorías presupuestales; teniendo muchos proyectos y actividades que no pudo concretar; o realizó avances pocos significativo que demostraron muchas deficiencias en todo el procedimiento de efectuación del gasto público. La Dirección Regional de Transportes y Comunicaciones de San Martín contó con un PIM de S/. 16878721 para el año 2019; el 
cual tuvo un $81.1 \%$ de avance de la ejecución presupuestal. Esto fue debido a que muchos proyectos estuvieron en $0 \%$, dando a entender que la calidad de gasto público no fue positiva en la aprobación, ejecución, eficacia, eficiencia y transparencia; ya que no estuvo siendo llevado a cabo de la manera adecuada, y con ello la gestión se vio encaminada hacia la deficiencia y la malversación (Ministerio de Economía y Finanzas, 2019).

En ese sentido, el objetivo de la presente investigación fue medir el nivel de la calidad de gasto público respecto al proceso presupuestario en la Dirección Regional de Transportes y Comunicaciones San Martin, 2019; con la finalidad de tomar medidas correctivas que coadyuven en el logro de los objetivos de la institución, así como contribuir en el aspecto teórico para otras investigaciones que requieran analizar los componentes de la calidad de gasto público y el proceso presupuestario a fin de mejorar la gestión de una entidad.

\section{ESTRATEGIAS METODOLÓGICAS O MATERIALES Y MÉTODOS}

La presente investigación tuvo un diseño no experimental, de tipo documental, donde consistió en revisar material bibliográfico seleccionado sobre el objeto de estudio para realizar un análisis sobre el contenido de los mismos (Corona y Maldonado, 2018). El cual fue necesario el uso de una serie de métodos y técnicas de búsqueda, procesamiento y almacenamiento de la información contenida en los documentos, en primera instancia, y la presentación sistemática, coherente y suficientemente argumentada de nueva información en el documento científico, en segunda instancia (Tancara, 1993).

En ese sentido, la investigación tuvo el siguiente diseño:

\section{$M \rightarrow X \rightarrow Y$}

\section{Donde:}

$\mathrm{M}=$ Documentos recopilados.

$\mathrm{X}=$ Información del gasto público del proceso presupuestal en la DRTC-SM, 2019.

$\mathrm{Y}=$ Conclusiones identificadas.

Como técnica se hizo uso del análisis documentario, abarcando un conglomerado de información de fuentes recopiladas del Sistema Integrado de Administración Financiera (SIAF-SP) y MEF - Portal de Transparencia Económica Consulta Amigable. Producto de ello se elaboraron fichas de recolección de datos como instrumento del estudio realizado. Los procedimientos que se realizaron para hacer realidad el estudio, consistió en obtener la autorización de los responsables de la Dirección Regional de Transportes y 
Comunicaciones de San Martin, entidad que fue objeto de la investigación. Seguidamente, para la extracción de la información se tuvo apoyo del Portal de Transparencia Económica Consulta Amigable del MEF, además se consolidó información relevante para el estudio; dicha información obtenida se procesó mediante el software Microsoft Excel, que sirvió para dar una mejor presentación de los datos en tablas y figuras, que ayudó en la interpretación de los resultados.

\section{RESULTADOS Y DISCUSIÓN}

3.1. Calidad de gasto publico respecto a la formulación del proceso presupuestario en la Dirección Regional de Transportes y Comunicaciones San Martin, 2019.

Tabla 1. Metas Presupuestales - Año Fiscal 2019

\begin{tabular}{|c|c|c|}
\hline $\begin{array}{l}\text { Meta } \\
\text { Física }\end{array}$ & Descripción & Monto (S/.) \\
\hline 4 & $\begin{array}{l}\text { Mantenimiento periódico de la red vial departamental no } \\
\text { pavimentada }\end{array}$ & 4985381.00 \\
\hline 14 & Abastecimiento de bienes y servicios & 3191962.00 \\
\hline 1 & $\begin{array}{l}\text { Mantenimiento periódico emp. pe 5n (Div. Cuñumbuque) - San } \\
\text { José de Sisa - Agua Blanca - San Pablo - emp. pe 5n (Div. } \\
\text { Bellavista) }\end{array}$ & 12 \\
\hline 2 & $\begin{array}{l}\text { Mantenimiento periódico de la carretera Dptal. ruta sm } 106 \\
\text { emp. pe 5n (Puente Colombia)- Shapaja Chazuta }\end{array}$ & 1000000.00 \\
\hline 23 & Mantenimiento y reparación de equipo mecánico & 725970.00 \\
\hline 26 & Mantenimiento a los sistemas de comunicación comunal & 634344.00 \\
\hline 29 & $\begin{array}{l}\text { Mantenimiento periódico de la red vial no pavimentada } \\
\text { carretera Dptal ruta sm 100, Jepelacio-Barranquita-Nuevo San } \\
\text { Miguel-Carrizales }\end{array}$ & 614000.00 \\
\hline 35 & $\begin{array}{l}\text { Mantenimiento periódico carretera Dptal. ruta sm } 116 \text { - tramo } \\
\text { San Roque de Cumbaza- Chunchihui- Urcopata-emp sm } \\
\text { 115(Div. Urcopata) }\end{array}$ & 608000.00 \\
\hline 28 & $\begin{array}{l}\text { Mantenimiento periódico de la red vial no pavimentada } \\
\text { carretera Dptal ruta sm 100, tramo: San Martin de Alao-Nuevo } \\
\text { Pucacaca-Constancia- Sinami }\end{array}$ & 607208.00 \\
\hline
\end{tabular}

Fuente: Datos obtenidos del SIAF-RP, 2019.

Se consolidaron las diez metas más significativas que presentaron montos importantes, el cual se rescataron 03 montos más grandes de la formulación presupuestal, teniendo la Meta 04: Mantenimiento periódico de la red vial departamental no pavimentada con un total de S/. 4985 381.00; la Meta 014: Abastecimiento de bienes y servicios la suma de S/. 3191962.00 y la Meta 01: Mantenimiento periódico emp. pe 5n (Div.Cuñumbuque) 
- San José de Sisa - Agua Blanca - San Pablo - Emp. pe 5n (Div.Bellavista) con el valor de S/. 1267 667.00, tal como se muestra en la (Tabla 1).

\subsection{Calidad de gasto publico respecto a la aprobación del proceso presupuestario} en la Dirección Regional de Transportes y Comunicaciones San Martin, 2019

Tabla 2. Presupuesto Institucional de Apertura por Fuente de Financiamiento

\begin{tabular}{lccccc}
\hline $\begin{array}{c}\text { Fuente de } \\
\text { Financiamiento }\end{array}$ & PIA (S/.) & PIM (S/.) & \multicolumn{2}{c}{ Ejecución (S/.) } & Avance \\
Devengado & Girado & \% \\
\hline $\begin{array}{l}\text { 1:Recursos } \\
\text { ordinarios }\end{array}$ & 11088090.00 & 12080575.00 & 11443343.00 & 11440994.00 & 94.7 \\
$\begin{array}{l}\text { 2:Recursos } \\
\text { directamente } \\
\text { recaudados }\end{array}$ & 3177288.00 & 4795008.00 & 2251383.00 & 2251151.00 & 47.0 \\
$\begin{array}{l}\text { 3:Recursos por } \\
\text { operaciones } \\
\text { oficiales de } \\
\text { crédito }\end{array}$ & 0.00 & 20,857 & 0.00 & 0.00 & 0.0 \\
\hline
\end{tabular}

Fuente: Datos obtenidos del SIAF-RP, 2019.

La fuente de financiamiento por recursos ordinarios (Presupuesto que asigna el Ministerio de Economía de Finanzas MEF) contó con un valor de PIA de S/ 11088090.00 y PIM de S/. 12080 575.00; que según el devengado se obtuvo un avance de 94.7\%. Por otra parte, la fuente de financiamiento de recursos directamente recaudados (recaudación que ingresa a la entidad) contó con un valor PIA de S/. 3177288.00 y PIM de S/. 4795 008.00; que presentó un avance de 47.0\%. Finalmente, se reportó que los recursos por operaciones oficiales de crédito tuvieron un valor PIA de S/. 0.00 y un PIM de S/. 20 857.00; que no presentó ningún avance, quedando este valor en $0.0 \%$.

Tabla 3. Presupuesto Institucional de Apertura por Genérica

\begin{tabular}{|c|c|c|c|c|c|c|}
\hline \multirow{2}{*}{\multicolumn{2}{|c|}{ Genérica }} & \multirow{2}{*}{ PIA (S/.) } & \multirow{2}{*}{ PIM (S/.) } & \multicolumn{2}{|c|}{ Ejecución (S/.) } & \multirow{2}{*}{$\begin{array}{c}\text { Avance } \\
\%\end{array}$} \\
\hline & & & & Devengado & Girado & \\
\hline $\begin{array}{l}\text { 5-22: Pensiones } \\
\quad \text { otras } \\
\quad \text { prestaciones } \\
\text { sociales }\end{array}$ & $\mathrm{y}$ & 508436.00 & 532721.00 & 501743.00 & 501270.00 & 94.2 \\
\hline $\begin{array}{l}\text { 5-21: } \text { Personal } \\
\text { obligaciones } \\
\text { sociales }\end{array}$ & $\mathrm{y}$ & 2510762.00 & 2775673.00 & 2521873.00 & 2520082.00 & 90.9 \\
\hline $\begin{array}{l}\text { 5-23:Bienes } \\
\text { servicios }\end{array}$ & $\mathrm{y}$ & 10956151.00 & 13090366.00 & 10335206.00 & 10334889.00 & 79.0 \\
\hline $\begin{array}{l}\text { 6-26: Adquisición } \\
\text { de activos } \\
\text { financieros }\end{array}$ & no & 290029.00 & 497680.00 & 335904.00 & 335904.00 & 67.5 \\
\hline
\end{tabular}

Fuente: Datos obtenidos del SIAF-RP, 2019. 
En cuanto, al Presupuesto Institucional de Apertura por Genérica (Tabla 3), la genérica 21: Personal y obligaciones sociales, que incluye a los trabajadores contratados y nombrados bajo el régimen laboral 276, la asignación del PIA fue de S/. 2510762.00 y el PIM de S/. 2775 673.00, cual tuvo un avance de 90.9\%; asimismo, la genérica 22: Pensiones y otras prestaciones sociales, es el presupuesto asignado para el pago de trabajadores jubilados, tuvo un PIA S/. 508436.00 y PIM de S/. 532 721.00, cual presentó un avance de 94.2\%; de esa manera, la genérica 23: Bienes y servicios que ejecuta la entidad en los gastos corrientes contó con un PIA de S/. 10956151.00 y PIM de S/. 13 090 366.00; ejecutándose según devengado el 79.0\% de avance; finalmente la genérica 26: Adquisición de activos no financieros, que son los gastos de capital su PIA fue de S/. 290029.00 y el PIM de S/. 497 680.00, que presentó un avance de 67.5\%.

\subsection{Calidad de gasto publico respecto a la ejecución del proceso presupuestario en}

la Dirección Regional de Transportes y Comunicaciones San Martin, 2019.

Tabla 4. Ejecución de Ingresos - Recursos Directamente Recaudados

\begin{tabular}{lr}
\hline \multicolumn{1}{c}{ Ingresos } & Total (S/.) \\
\hline Venta de bases para licitación pública, concurso público y otros & 72.20 \\
Tramite por tránsito vehicular & 845.00 \\
Derechos de permiso de operación & 209585.70 \\
Derechos de inscripción & 77119.20 \\
Derechos de examen & 424966.90 \\
Derechos de comunicaciones y telecomunicaciones & 0.00 \\
Licencias & 379723.60 \\
Expedición de constancias & 2101.50 \\
Otros derechos administrativos de transportes y comunicaciones & 17067.30 \\
Otros derechos administrativos & 831.10 \\
Servicio de capacitación & 2982.40 \\
Intereses por depósitos distintos de recursos por privatización y & 11345.53 \\
concesiones & por \\
Derecho por uso del espectro radioeléctrico - canon & 1603928.00 \\
telecomunicaciones & 347302.45 \\
Infracciones de reglamento de transporte & 13945.50 \\
Otras multas & 186.00 \\
Otros ingresos diversos & $\mathbf{9 3 0 . 1 8}$ \\
Total & $\mathbf{3 0 9 1}$ \\
\hline
\end{tabular}

Fuente: Datos obtenidos del SIAF-RP, 2019.

En la tabla 4, referente a la ejecución de ingresos por parte de la entidad en diversos conceptos, se resaltaron los montos más significativos, siendo el derecho por uso del espectro radioeléctrico - canon por telecomunicaciones con el valor de S/ 1603 928.00, 
otro monto significativo se consideró a los ingresos por derecho de examen con la suma de S/ 424 966.90, también la recaudación por tramite de licencias con el total de S/ 379 723.60, además el ingreso por infracciones de reglamento de transporte contó con la recaudación de S/ 347302.45 y por último el monto por derecho de permiso de operación la suma de S/ 209585.70.

Tabla 5. Ejecución de gastos - Recursos Ordinarios

\begin{tabular}{lccccc}
\hline Genérica & PIM (S/.) & Devengado (S/.) & Diferencia (S/.) & Avance \% \\
\hline $\begin{array}{l}\text { 5-21:Personal y } \\
\text { obligaciones sociales }\end{array}$ & 2775673.00 & 2521873.00 & 253800.00 & 90.90 \\
$\begin{array}{l}\text { 5-22:Pensiones y otras } \\
\text { prestaciones sociales }\end{array}$ & 532721.00 & 501743.00 & 30978.00 & 94.20 \\
$\begin{array}{l}\text { 5-23: Bienes y servicios } \\
\text { 6-26:Adquisición de }\end{array}$ & 2562693.00 & 8212126.00 & 350567.00 & 95.90 \\
$\quad \begin{array}{l}\text { activos no financieros } \\
\text { Total }\end{array}$ & $\mathbf{1 2 ~ 0 8 0 ~ 5 7 5 . 0 0}$ & $\mathbf{1 1 ~ 4 4 3 ~ 3 4 4 . 0 0}$ & $\mathbf{6 3 7 ~ 2 3 1 . 0 0}$ & $\mathbf{9 5 . 0 3}$ \\
\hline
\end{tabular}

Fuente: Datos obtenidos del SIAF-RP, 2019.

Con respecto a la ejecución de gastos por recursos ordinarios, se observó 04 genéricas de gasto (Tabla 5). La genérica 21: Personal y obligaciones sociales tuvo un avance de 90.90\% con un presupuesto que no se devengó de S/ 253 800.00; asimismo, la genérica 22: Pensiones y otras prestaciones sociales contó con un avance de $94.20 \%$ ya que no se devengó el total de S/ 30 978.00; de la misma manera la genérica 23: Bienes y servicios obtuvo un avance de $95.90 \%$ teniendo una diferencia que no se devengó de S/ 350567.00 y por último la genérica 26:Adquisición de activos no financieros reportó un avance de $99.10 \%$ siendo el monto no devengado de S/ 1 886.00. Por lo tanto, en general no se devengó un total de S/ 637 231.00, monto que fue revertido al tesoro público del Ministerio de Economía y Finanzas - MEF.

Tabla 6. Ejecución de gastos - Recursos Directamente Recaudados

\begin{tabular}{lrlll}
\multicolumn{1}{c}{ Genérica } & PIM (S/.) & \multicolumn{1}{c}{ Devengado (S/.) } & Diferencia (S/.) & $\begin{array}{c}\text { Avance } \\
\text { \% }\end{array}$ \\
\hline $\begin{array}{l}\text { 5-23: Bienes y servicios } \\
\text { 6-26: Adquisición de }\end{array}$ & 267335.00 & 2123080.00 & 2404593.00 & 46.90 \\
$\begin{array}{l}\text { activos no financieros } \\
\text { Total }\end{array}$ & $\mathbf{4 7 9 5 0 0 8 . 0 0}$ & $\mathbf{2 2 5 1 3 8 2 . 0 0}$ & $\mathbf{2 5 4 3 6 2 6 . 0 0}$ & $\mathbf{4 7 . 4 5}$ \\
\hline
\end{tabular}

Fuente: Datos obtenidos del SIAF-RP, 2019.

En la tabla 6, se pudo apreciar la ejecución de gastos por recursos directamente recaudados, donde la genérica 23: Bienes y servicios tuvo un avance de $46.90 \%$ ya que 
no se ejecutó el monto de S/ 2404 593.00, por otra parte, la genérica 26: Adquisición de activos no financieros reportó un avance de $48.00 \%$ con un presupuesto que no se ejecutó de S/ 139 033.00. Por lo tanto, el total de S/ 2543626.00 no fue devengado, dicho saldo es revertido para el presupuesto institucional de apertura - PIA para el siguiente año fiscal.

Tabla 7. Ejecución de gastos - Recursos por operaciones oficiales de crédito

\begin{tabular}{lccc}
\hline \multicolumn{1}{c}{ Genérica } & PIM & Devengado & Avance \% \\
\hline 6-26:Adquisición de activos no financieros & 20857.00 & 0.00 & 0.00 \\
Total & $\mathbf{2 0 ~ 8 5 7 . 0 0}$ & $\mathbf{0 . 0 0}$ & 0.00 \\
\hline
\end{tabular}

Fuente: Datos obtenidos del SIAF-RP, 2019.

Adicionalmente, se reportó que la ejecución de gastos de recursos por operaciones de crédito no presento ningún avance respecto al PIM de S/ 20 857.00, esto perjudicó a la entidad para medir el nivel de gasto público (Tabla 7).

3.4. Calidad de gasto público respecto al proceso presupuestario en la Dirección Regional de Transportes y Comunicaciones San Martin, 2019

Tabla 8. Ejecución general de genéricas de gastos

\begin{tabular}{lcccc}
\hline Genérica de gastos & PIM & Devengado & Diferencia & Avance \% \\
\hline $\begin{array}{c}\text { 5-21:Personal } \\
\text { obligaciones sociales }\end{array}$ & 2775673.00 & 2521873.00 & 253800.00 & 90.90 \\
$\begin{array}{c}\text { 5-22:Pensiones y otras } \\
\text { prestaciones sociales }\end{array}$ & 532721.00 & 501743.00 & 30978.00 & 94.20 \\
$\begin{array}{c}\text { 5-23: Bienes y servicios } \\
\text { 6-26:Adquisición de activos } \\
\text { no financieros }\end{array}$ & 43090366.00 & 10335206.00 & 2755160.00 & 79.00 \\
\begin{tabular}{c} 
Total \\
\hline
\end{tabular} & $\mathbf{1 6 8 9 6 4 4 0 . 0 0}$ & $\mathbf{1 3 6 9 4 7 2 6 . 0 0}$ & $\mathbf{3 2 0 1 7 1 4 . 0 0}$ & $\mathbf{8 1 . 1 0}$ \\
\hline
\end{tabular}

Fuente: Datos obtenidos del SIAF-RP, 2019

La información sobre la ejecución de gastos se observa en la tabla 8, en base a eso se calculó la calidad en gasto teniendo en cuenta la escala señalada en la tabla 9.

Tabla 9. Escala de calidad de gastos

\begin{tabular}{lc}
\hline Nivel de calidad de gasto & Calificación \\
\hline Muy bueno & $95 \%-100 \%$ \\
Bueno & $90 \%-94 \%$ \\
Regular & $85 \%-89 \%$ \\
Deficiente & $00 \%-84 \%$ \\
\hline
\end{tabular}

Fuente: Directiva No 005-2012-EF/50.01, 2012 - Ministerio de Economía y Finanzas 
En la tabla 9, se pudo apreciar que la genérica de gastos con mayor avance fue la 22: Pensiones y otras prestaciones sociales, el cual tuvo una ejecución de $94.20 \%$, debido que no se devengó la suma de S/ 30 978.00; por lo tanto, el nivel de calidad de gasto según la tabla de la directiva se considera como "Bueno"; asimismo, la genérica 21: Personal y obligaciones sociales, a pesar que tuvo un saldo de S/ 253800.00 que no se devengó, se cumplió el 90.90\% de ejecución; donde el nivel de calidad de gasto ejecutado fue "Bueno". De la misma forma, la genérica de gastos 23: Bienes y servicios, tuvo un saldo diferencial de S/ 2755 160.00, con un avance presentado del 79.00\%, el cual se categorizó como ejecución "Deficiente". Finalmente, la genérica 26: Adquisición de activos no financieros presentó un saldo diferencial de S/ 161776.00 con un avance del 67.50\% que al igual que la genérica anterior se ubican como ejecución "Deficiente". En general la Dirección Regional de Transportes y Comunicaciones de San Martín durante el año fiscal 2019 tuvo una ejecución del presupuesto del 81.1\% el cual según tabla de calificación la calidad de gasto fue "Deficiente".

\section{DISCUSIÓN}

El estudio tuvo como objetivo medir el nivel de la calidad de gasto público respecto al proceso presupuestario en la Dirección Regional de Transportes y Comunicaciones de San Martin durante el año 2019, el cual, según información analizada dentro del estudio, se evaluó y se calificó como calidad de gasto deficiente, ya que la ejecución que tuvo fue de $81.10 \%$ y según escala valorativa de la Directiva $N^{\circ} 005$-2012-EF/50.01, 2012 del Ministerio de Economía y Finanzas, se pudo calificar dicho gasto público en ese nivel. Esta afirmación se respalda tomando referencia a los autores como Rodríguez et al. (2020), en su estudio concluye en relación al nivel de gasto público, donde el $87.5 \%$ de los trabajadores y $80 \%$ de los funcionarios percibe que el nivel de gasto público es "Bajo", debido a que no es eficiente, eficaz y transparente su ejecución. Asimismo, el gasto público se caracteriza porque "Nunca o casi nunca", se gestiona el cumplimiento de los objetivos institucionales de manera eficiente, aprobación y recepción de los informes de trabajo en los plazos establecidos, evaluación de los resultados para el logro de las metas, planificación del presupuesto de acuerdo a los documentos normativos. Asimismo, en esa misma linea Guerra y Palomino (2020), muestra claras evidencias de procesos deficientes en la ejecución de gasto en la institución estudiada, estos no solo en la percepción de los diversos especialistas, sino también en los que conforman dichas unidades como son los 
directores. Otro estudio va relacionado con lo manifestado por Yactayo (2019), según los resultados encontrados refleja que la ejecución presupuestal de los años 2008 al 2013 en una institución de Salud del Perú, no son satisfactorios para los asegurados, no obstante la entidad gasta al $100 \%$ de lo presupuestado, pues hay deficiencia en el aprovisionamiento de material estratégico y en la calidad de atención al paciente asegurado, así como falta de médicos especializados entre otras carencias. El resultado también se asemeja a realidades extranjeras vecinas donde se tiene a Bolivia en un estudio realizado por Molina y Gantier (2017), que manifiesta tanto para el periodo 1990-2015 como para el periodo 1990-2004 evidenciándose un multiplicador del gasto negativo en dicho país. No obstante, para el periodo 2005-2015, se evidencia un multiplicador positivo, demostrando así que el gasto de gobierno es una herramienta importante para el crecimiento de la economía boliviana. También es el caso de México donde Campo y Mendoza (2018), exponen la existencia de inefectividad de los diferentes gobiernos como resultado de la corrupción rampante, la incompetencia, el clientelismo y el burocratismo; afecta a la calidad de gasto público en dicha nación. Así como la inconducta funcional, la falta de ética, la falta de capacidad de gestión, etc., existen otros factores que afectan a la calidad de gasto público en un país, es así que se tiene a la gestión organizacional como factor clave en una buena ejecución presupuestal, según Navarro y Delgado (2020), manifiesta que la gestión organizacional es importante, porque el comportamiento de las personas depende de ello y es necesario que se establezca funciones bien definidas para ejercer el cargo. Asimismo, las consecuencias institucionales principalmente por una deficiente gestión organizacional van en incremento y conlleva a una baja ejecución presupuestal municipal. Otro componente está relacionado con el sistema integrado de administración financiera donde Valera y Delgado (2020) indica que un sistema integrado de administración financiera radica en los principios de objetividad, sencillez, transparencia, suficiencia financiera, equidad, solidaridad, autonomía, corresponsabilidad, coordinación, lealtad institucional y financiera para el manejo y uso de los recursos del Estado, además atribuido al control administrativo y financiero con competencias integrales para el uso y disposición de los recursos públicos se verá reflejado en una eficiente y eficaz manejo de los recursos asignados por el Estado. También cabe indicar que la planificación es elemento clave para una buena ejecución presupuestal donde Nuñez et al. (2018), precisa que la planificación del desarrollo 
económico y social de la Nación, repercute en la buena gestión presupuestaria y en su eficiente ejecución, lo que implica lograr alcanzar los objetivos institucionales de la forma más eficiente. De igual forma el control y la evaluación es crucial en el proceso presupuestario que según Coronel et al. (2020), alude que la institución que no disponen de mecanismos de control y de evaluación constante a las fases del ciclo presupuestario, en torno a ello estará condenado a la ineficiencia en la ejecución del presupuesto asignado en el periodo evaluado. Finalmente Faroni et al. (2017), refiere que debe realizarse una verdadera planificación y elaboración presupuestaria, siendo de esa manera que los gobernantes y los legisladores podrán, en esencia, promover una mayor calidad de gasto y transparencia de la gestión.

\section{CONCLUSIÓN O CONSIDERACIONES FINALES}

\section{En conclusión, podemos manifestar que:}

1. Se identificó que la calidad de gasto público respecto al proceso presupuestario en la Dirección Regional de Transportes y Comunicaciones San Martin, 2019, fue calificada como deficiente, debido que el avance de ejecución del proceso presupuestario fue de $81.1 \%$.

2. Durante el año fiscal 2019 la entidad obtuvo en la formulación del proceso presupuestario, 51 metas presupuestales aprobadas, teniendo las 10 metas más significativas que se menciona en la tabla 1 para la ejecución presupuestal.

3. En la aprobación del proceso presupuestario estuvo dada por la fuente de financiamiento Recursos Ordinarios con un PIA de S/ 11088 090.00 y PIM de S/. 12 080 575.00; los Recursos Directamente Recaudados con un PIA de S/. 3177288.00 y PIM de S/. 4795008.00 y por último los recursos por operaciones oficiales de crédito con un valor PIA de S/. 0.00 y un PIM de S/. 20,857.00; cual tuvo como suma total un PIA total de S/. 14265378.00 y un PIM total de S/. 16896440.00.

4. Finalmente como parte de la ejecución de ingresos por los Recursos Directamente Recaudados durante el periodo fiscal 2019 se recaudó S/. 3091 930.18. Por otro lado, respecto a la ejecución de gastos por Recursos Ordinarios se devengó S/. 11443 344.00 cual tuvo un avance de 95.03\%; asimismo, referente a la ejecución gastos por Recursos Directamente Recaudados se devengó un total de S/. 2251382.00 y el avance fue de $47.45 \%$; por último, la ejecución de gastos por Recursos por 
operaciones oficiales de Crédito fue de $0.00 \%$ y esto es en relación a la ejecución del proceso presupuestario.

\section{LISTA DE REFERENCIAS}

Campo, J., \& Mendoza, H. (2018). Gasto público y crecimiento económico: un análisis regional para Colombia, 1984-2012. Lecturas de Economía, 88, 77-108. https://doi.org/10.17533/udea.le.n88a03 Primera

Carmen Moran, C. (2019). Pésima ejecución presupuestal 2019. 12 Nov 2019. https://www.expreso.com.pe/destacado-portada/pesima-ejecucion-presupuestal$2019 /$

Comisión Económica para América Latina y el Caribe. (2002). El sistema presupuestario $\begin{array}{lllll}\text { en el Perú (R. Mostajo } & \text { (ed.)). }\end{array}$ https://www.cepal.org/sites/default/files/publication/files/7267/S023177_es.pdf

Corona, J., \& Maldonado, J. (2018). Investigación Cualitativa: Enfoque Emic-Etic. Revista Cubana de Investigaciones Biomédicas, 37(4), 1-4. http://scielo.sld.cu/pdf/ibi/v37n4/ibi22418.pdf

Coronel, E., Narváez, C., \& Erazo, J. (2020). Evaluation of the planning process and the budget cycle for Decentralized Autonomous Governments. Revista Arbitrada Interdisciplinaria Koinonía, 5(10), 1-28. https://doi.org/10.35381/r.k.v5i10.688

Faroni, W., Da Silva, M., \& Diogo, A. (2017). Budgetary planning instruments: potentialities and weaknesses in the process. Revista de Economía Crítica, 23, 8295. https://dialnet.unirioja.es/servlet/articulo?codigo=6247399

Galiya, A., Malika, K., Kuanyshbek, A., Salima, S., \& Gulmira, B. (2018). Managerial competence of future specialists of the education system (Preschool education and upbringing) and medicine in the comparative aspect. Opción, 34(85), 44-62. https://www.redalyc.org/articulo.oa?id=31055914002\%0AHow

Guerra, I., \& Palomino, G. del P. (2020). Modelo de gestión por resultados para mejorar la calidad de gasto en la Unidad de Gestión Educativa Local, San Martín. Ciencia Latina Revista Multidisciplinar Multidisciplinar, 4(2), 738-758. https://doi.org/10.37811/cl_rcm.v4i2.112

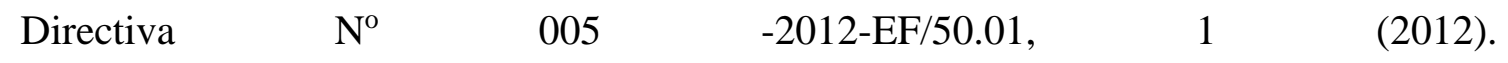

https://www.mef.gob.pe/contenidos/presu_publ/anexos/Directiva_005_2018EF5 001.pdf 
Ministerio de Economía y Finanzas. (2019). Seguimiento de la Ejecución Presupuestal (Consulta amigable). https://apps5.mineco.gob.pe/transparencia/Navegador/default.aspx

Ministerio de Economía y Finanzas. (2020). Resumen ejecutivo de la ejecución del gasto de los Programas Presupuestales. https://www.mef.gob.pe/contenidos/presu_publ/ppr/Reporte_Anual_2019_VCA _17_04_19.pdf

Molina, G., \& Gantier, M. (2017). El gasto público y su efecto en la economía Boliviana: periodo 1990-2015. Universidad Católica Boliviana, 20(39), 7-42. http://www.scielo.org.bo/scielo.php?script=sci_arttext\&pid=S199437332017000100002

Navarro, K., \& Delgado, J. (2020). Gestión organizacional en la ejecución presupuestal Municipal 2020. Ciencia Latina Revista Multidisciplinar Multidisciplinar, 4(2), 1053-1071. https://doi.org/10.37811/cl_rcm.v4i2.138

Nuñez, L., Bravo, L., Cruz, C., \& Hinostroza, M. del C. (2018). Competencias gerenciales y competencias profesionales en la gestión presupuestaria. Revista Venezolana de Gerencia, 23(83). https://www.redalyc.org/jatsRepo/290/29058775015/29058775015.pdf

Rodríguez, M., Palomino, G. del P., \& Aguilar, C. (2020). Eficiencia , eficacia y transparencia del gasto público municipal Efficiency, effectiveness and transparency of municipal public. Ciencia Latina Revista Multidisciplinar Multidisciplinar, 4(2), 704-719. https://doi.org/10.37811/cl_rcm.v4i2.108

Schröder, P. (2013). Nueva Gestión Pública: Aportes para el buen gobierno (Fundación Friedrich Naumann (ed.)). https://relial.org/uploads/biblioteca/44dbee76837e79a6c07bb8219d021843.pdf

Shack, N., Pérez, J., \& Portugal, L. (2020). Cálculo del tamaño de la corrupción y la inconducta funcional en el Perú. https://doc.contraloria.gob.pe/estudiosespeciales/documento_trabajo/2020/Calculo_de_la_Corrupcion_en_el_Peru.pdf

Tancara, C. (1993). La Investigación documental. Temas Sociales, 17, 91-106. http://www.scielo.org.bo/scielo.php?script=sci_arttext\&pid=S004029151993000100008 
Valera, J., \& Delgado, J. (2020). Control interno de la ejecución presupuestal en la gestión de salud 2020. Ciencia Latina Revista Multidisciplinar, 4(2), 1092-1110. https://doi.org/10.37811/cl_rcm.v4i2.140

Vargas, J., \& Zavaleta, W. (2020). La gestión del presupuesto por resultados y la calidad del gasto en gobiernos locales. Visión de Futuro, 24(2), 37-59. https://doi.org/10.36995/j.visiondefuturo.2020.24.02.002.es

Yactayo, E. (2019). La ejecución presupuestal de ESSALUD del Perú como un instrumento de gestión. Pensamiento Crítico, 24(1), 103-120. https://doi.org/10.15381/pc.v24i1.16561 